José Florencio F. Lapeña, Jr. MA, MD

Department of Otorhinolaryngology College of Medicine, University of the Philippines Manila, Philippines

Department of Otorhinolaryngology Head and Neck Surgery

East Avenue Medical Center, Diliman

Quezon City, Philippines

\section{Authorship Controversies: Gift, Guest and Ghost Authorship}

Authorship, "the state or fact of being the writer of a book, article, or document, or the creator of a work of art,"1 derives from the word author, auctor, autour, autor "father, creator, one who brings about, one who makes or creates," from Old French auctor, acteor "author, originator, creator, instigator," directly from the Latin auctor "promoter, doer; responsible person, teacher," literally "one who causes to grow."2 It implies a creative privilege and responsibility that cannot be taken lightly. In the biomedical arena, the International Committee of Medical Journal Editors (ICMJE) "recommends that authorship be based on the following four criteria: 1. Substantial contributions to the conception or design of the work; or the acquisition, analysis, or interpretation of data for the work; AND 2. Drafting the work or revising it critically for important intellectual content; AND 3. Final approval of the version to be published; AND 4. Agreement to be accountable for all aspects of the work in ensuring that questions related to the accuracy and integrity of any part of the work are appropriately investigated and resolved." ${ }^{\prime 3}$

Thus, all persons designated as authors should qualify for authorship, and all those who qualify as authors should be so listed. ${ }^{3}$ The first of these general principles means that all persons listed as authors should meet the four ICMJE criteria for authorship; the second principle means that all those who meet the four ICMJE criteria for authorship should be listed as authors. ${ }^{3}$ The first part of the statement disqualifies honorific "gift" authors, complementary "guest" authors and anonymous "ghost" authors from being listed as authors. The second part ensures the listing of all those who qualify as authors, even if they are no longer part of the institution or group from which the work emanates (such as students who have graduated or residents and fellows who have completed their postgraduate training).

Honorific or "gift" authorship takes place when a subordinate (or junior) person lists a superior (or senior) person as an author, even if that person did not meet the four ICMJE authorship criteria. ${ }^{4,5}$ Bestowing the gift on a Chief, Chair, Department Head, Director, Dean, or such other person is often done in gratitude, but carries an unspoken expectation that the favor will be returned in the future. It can also be bestowed under coercive conditions (that may overlap with those of guest authorship discussed next). ${ }^{4.5} \mathrm{It}$ is unethical because the gifted person does not qualify for authorship when at most only acknowledgement is his or her due. In the extreme, such a person can be put in the uncomfortable and embarrassing situation of being unable to comment on the supposedly co-authored work when asked to do so. Moreover, the unqualified co-author(s) may actually attempt to wash their hands of any allegations of misconduct, claiming for example that the resident first author "plagiarized the material" or "fabricated or manipulated the data" but "I/we certainly had nothing to do with that" - - hence the fourth criterion for authorship came to be. ${ }^{3}$ Reviewers and Editors may suspect "gift" authorship when 
for instance, a resident listed as first author writes the paper in the first person, using the pronoun "I" instead of "we" and thanks the consultant co-author under the "acknowledgements" section. The suspicions are further reinforced when the concerned co-author(s) do not participate in (or contribute to) revising the manuscript critically for important intellectual content during the review and editing process.

Guest authorship takes place when influential or well-known individuals "lend" their name to a manuscript to boost its prestige, even though they had nothing to do with its creation. ${ }^{6,7}$ They may have been invited to do so by one or more of the actual authors, but they willingly agree, considering the arrangement mutually-beneficial. Thus, a student or resident may knowingly invite an adviser or consultant to be listed as co-author, even if the latter did not meet authorship criteria. The former perceives that having a known co-author increases the chances of a favorable review and publication; the latter effectively adds another publication to his or her curriculum vitae. It is not difficult to see how such symbioses may thrive in the "publish or perish" milieu of academe. Research advising alone, even if editing of the research paper was performed, do not qualify one for authorship (cf. "gift" authorship). This is not to say that a research, thesis or dissertation adviser may not be listed as co-author - as long as he or she meets the 4 ICMJE criteria for authorship. ${ }^{3}$ A related misconduct is the practice by certain persons with seniority of insisting their names be listed first, even if more junior scholars did all the innovative thinking and research on a project. Indeed, the order of authorship can be a source of unhappiness and dispute. Authors should be listed in the order of their contributions to the work - the one who contributed most is listed first, and the order of listing should be a joint decision of all co-authors at the start of the study (reviewed periodically).

Ghost authorship usually pertains to paid professional writers who anonymously produce material that is officially attributed to another author. ${ }^{7,8}$ They may operate out of establishments that manufacture term papers, theses and dissertations for the right price (such as the infamous C.M. Recto district in downtown Manila, now replaced by numerous online services). They may also be employed by the pharmaceutical industry to write promotional, favorable studies that will list wellknown persons (professors, scientists, senior clinicians) as authors, often with consent and adequate compensation. ${ }^{8}$ Examples include "a professor at the University of Wisconsin" being paid "\$1,500 in return for putting his name" on "an article on the 'therapeutic effects' of their diet pill Redux (dexfenfluramine)," that was "pulled from the market" a year later "as doctors began reporting heart-valve injuries in as many as onethird of patients taking the drug" and the drug "later linked to dozens of deaths." Similar cases involved the "deadly drug" rofecoxib (Vioxx) "eventually blamed for some $60,000+$ deaths," that "was also linked to a number of shameful scandals relating to fraudulent studies and the use of ghostwriters to boost sales. ${ }^{\prime \prime}$ The costs involved are not meager; a pharmaceutical paid "a medical education communication company (MECC) to write articles in support of the drug" Neurontin (gabapentin) "to the tune of $\$ 13,000$ to $\$ 18,000$ per article. In turn, MECC paid $\$ 1,000$ each to friendly physicians and pharmacists to sign off as authors of the articles." $^{\prime \prime}$ Another pharmaceutical (that acquired Neurontin form the first pharmaceutical) "was found guilty of illegally promoting off-label uses of Neurontin," and "fined more than \$142 million in damages." Whether or not morbidities or mortalities ensue from the practice, both ghosts and beneficiary-authors should be held liable in such situations.

Clearly, the practice of "gift," "guest," and "ghost" authorship should not be entertained by authors or tolerated by editors and reviewers. Authorship should be based on the ICMJE authorship criteria. Our editors and reviewers vigilantly strive to uphold and protect the rights and welfare of our authors and the integrity and soundness of their research. We call on all fellows, diplomates and residents in training to do the same.

\footnotetext{
REFERENCES

1. Google Online Dictionary: "Authorship" [Cited 2019 June 5]. Available from: https://www. google.com/search?client=safari\&rls=en\&q=Dictionary\#dobs=authorship.

2. Online Etymology Dictionary: "author" [Cited 2019 June 5]. Available from: https://www. etymonline.com/word/author.

3. International Committee of Medical Journal Editors. Recommendations for the Conduct, Reporting, Editing, and Publication of Scholarly Work in Medical Journals. Updated December, 2018. [Cited 2019 June 5]. Available from: http://www.icmje.org/recommendations/browse/ roles-and-responsibilities/defining-the-role-of-authors-and-contributors.html\#two.

4. Smith J. Gift authorship: a poisoned chalice? BMJ 1994 Dec 3;309(6967):1456-57. doi: https:// doi.org/10.1136/bmj.309.6967.1456 PMID:7804037 PMCID: PMC2541639.

5. Zaki SA. Gift authorship - A cause for concern. Lung India. 2011 Jul-Sep;28(3):232-233. doi: 10.4103/0970-2113.83994 PMID: 21886969 PMCID: PMC3162772.
}

6. Harvey LA. Gift, honorary or quest authorship. Spinal Cord. 2018 Feb;56(2):91. doi: 10.1038/ s41393-017-0057-8. PMID:29422533.

7. Vera-Badillo FE, Napoleone M, Krzyzanowska MK, Albhai SM, Chan AW, Ocana A, et al. Honorary and ghost authorship in reports of randomized clinical trials in oncology. Eur J Cancer. 2016 Oct;66:1-8. doi: 10.1016/j.ejca.2016.06.023 PMID:27500368.

8. DeTora LM, Carey MA, Toroser D, Baum EZ. Ghostwriting in biomedicine: a review of the published literature. Curr Med Res Opin. 2019 May 22:1-9. doi: 10.1080/03007995.2019.1608101. PMID:30986084.

9. Mercola J. How big pharma fools even your doctor. [Cited 2019 Jan 08]. Available from: https:// articles.mercola.com/sites/articles/archive/2011/11/26/medical-journals-using-ghost-writers. aspx. 